\title{
Caractérisation du climat de vagues le long des côtes de France métropolitaine: variabilité et tendances à long-terme
}

\author{
Guillaume DODET ${ }^{1}$, Xavier BERTIN ${ }^{2}$, Frédéric BOUCHETTE ${ }^{3}$, \\ Fabrice ARDHUIN ${ }^{4}$, Bruno CASTELLE ${ }^{5}$, Romain CHAILAN ${ }^{6}$
}

1. UMR 6554 LETG, Université de Bretagne Occidentale, CNRS, IUEM, rue Dumont d'Urville, 29280 Plouzané, France.

guillaume.dodet@univ-brest.fr

2. UMR 7266 LIENSS, CNRS, Université de La Rochelle, Institut Littoral et

Environnement, 2 rue Olympe de Gouges, 17000 La Rochelle, France.

xavier.bertin@univ-Ir.fr

3. UMR 5243 Géosciences Montpellier, CNRS, Université de Montpellier, campus

Triolet, place Eugène Bataillon, 34095 Montpellier, France.

frederic.bouchette@gmail.com

4. Univ. Brest, CNRS, IRD, Ifremer, Laboratoire d'Océanographie Physique et Spatiale (LOPS), IUEM, Rue Dumont d'Urville, 29280 Plouzané, France.

ardhuin@ifremer.fr

5. UMR 5805 EPOC, CNRS, Université de Bordeaux, Allée Geoffroy Saint-Hilaire, 33615 Pessac, France. bruno.castelle@u-bordeaux.fr

6. IMAG, Université de Montpellier, CNRS, 34000 Montpellier, France.

romain.chailan@gmail.com

\section{Résumé :}

Cette étude propose de caractériser le climat de vagues le long des côtes de France métropolitaine à partir de sorties de modèles à haute résolution. Les observations issues du réseau national CANDHIS sont tout d'abord comparées aux sorties de modèles. Ces comparaisons révèlent une bonne capacité des modèles à reproduire les paramètres de vagues observés, avec toutefois des erreurs plus marquées en Méditerranée. Les valeurs moyennes annuelles et saisonnières des paramètres de vagues le long du littoral présentent une variabilité spatiale très marquée, qui dépend de l'exposition des côtes aux houles océaniques, de la présence d'îles à proximité des côtes et des forçages atmosphériques contrôlant les mers de vent. Finalement, les tendances des moyennes hivernales de hauteurs de vagues sont calculées sur la période 1994-2012. Ces tendances s'expliquent essentiellement par les fluctuations pluriannuelles de la circulation atmosphérique dans l'Atlantique Nord.

Mots-clés :

Climat de vagues, Variabilité saisonnière, Tendances pluriannuelles, Indices climatiques. 


\section{Thème 1 - Hydrodynamique côtière}

\section{Introduction}

Avec $5853 \mathrm{~km}$ de trait de côte le long de la Mer du Nord, de la Manche, de l'océan Atlantique et de la mer Méditerranée, le littoral de France métropolitaine est exposé à une large gamme de régimes de vagues. Or notre capacité à aménager ce littoral, prévenir les risques de submersion et d'érosion, prévoir les évolutions morphologiques ou encore exploiter l'énergie des vagues, dépend de notre compréhension du climat de vagues en zone côtière. Pour cela, les bases de données issues de simulations numériques rétrospectives permettent de compléter les observations in-situ et de caractériser précisément la variabilité des paramètres de vagues le long des côtes françaises. BENOIT \& LAFON (2004) ont ainsi réalisé un atlas des vagues le long des côtes françaises, hors Méditerranée, à partir d'un rejeu de 25 ans (1979-2003) généré avec le modèle spectral TOMAWAC (BENOIT et al., 1996). Ils ont ainsi pu déterminer les hauteurs de vagues extrêmes associées à une période de retour de 50 ans. Ces résultats ont été complétés par BERTIN \& DODET (2010) qui ont réalisé des simulations rétrospectives de 56 ans (1953-2009) dans le Golfe de Gascogne à partir du modèle spectral WAVEWATCH III (WW3, TOLMAN et al., 2014). Ces résultats de modèle ont notamment permis d'étudier l'influence de l'Oscillation Nord Atlantique (ONA) sur les tendances à long terme des hauteurs, périodes et directions des vagues. Dans cette étude, nous utilisons les résultats de deux simulations rétrospectives à haute résolution réalisées sur la façade Atlantique (incluant Mer du Nord et Manche) et sur la façade Méditerranéenne, afin de comparer les performances des modèles dans ces régions et de caractériser le climat de vagues, en terme de moyennes annuelles et saisonnières, le long de l'ensemble des côtes de France métropolitaine.

\section{Méthodes}

Afin d'étudier la variabilité du climat de vagues le long des côtes françaises, nous avons exploité les résultats de simulations rétrospectives (1994-2012) sur les façades Atlantique et Méditerranéenne (figure 1). Ces résultats ont été obtenus à partir du modèle spectral WW3 V4.18 (ROLAND \& ARDHUIN 2014) implémenté sur des grilles non-structurées et raffinées à la côte, permettant ainsi de bien reproduire l'évolution du champ de vagues en zone côtière (figure 2). Pour la façade Atlantique, nous avons utilisé le rejeu HOMERE (BOUDIERE et al., 2013) qui couvre le Golfe de Gascogne et la Manche. Ce modèle est forcé par les champs de vent issus de la réanalyse CFSR d'une résolution allant de $0.25^{\circ}$ à $0.5^{\circ}$ (SAHA et al., 2010) et par les courants de surface générés à partir d'un atlas de constituants harmoniques de marée obtenus à partir de sorties du modèle de circulation MARS (LAZURE \& DUMAS, 2008). Pour la façade Méditerranéenne, nous avons utilisé le rejeu MEGAGOL (CHAILAN, 2015) qui s'étend du Détroit de Gibraltar jusqu'au sud de l'Italie. Ce modèle est forcé par les champs de vents issus de la réanalyse ARPERA d'une 


\section{XVèmes Journées Nationales Génie Côtier - Génie Civil \\ La Rochelle, 29 au 31 mai 2018}

résolution de $50 \mathrm{~km}$ (HERRMANN \& SOMOT, 2008) et par les courants de surface issus de la réanalyse NEMOMed8 (BEUVIER et al., 2010) d'une résolution de $10 \mathrm{~km}$.

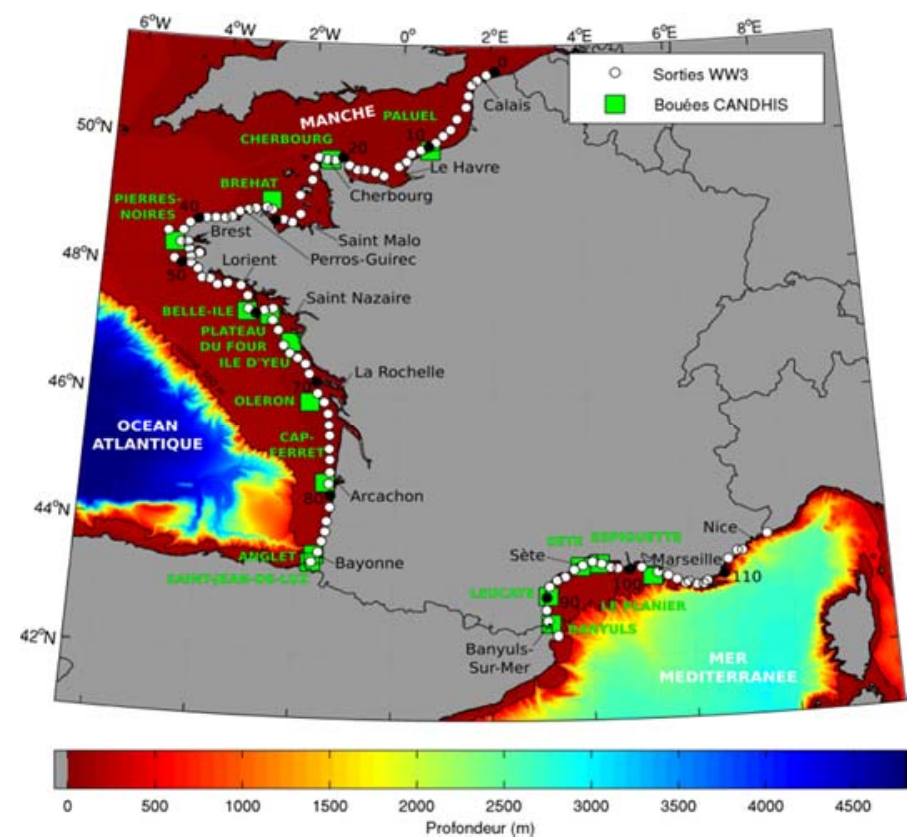

Figure 1. Carte bathymétrique et localisation des sorties de modèles (ronds blancs) et des bouées utilisées pour la validation des modèles (carrés verts).

Pour cette étude, nous avons uniquement utilisé les données disponibles sur la période de simulation commune à ces deux rejeux, c'est-à-dire de janvier 1994 à décembre 2012. Pour obtenir plus d'informations concernant l'implémentation et la paramétrisation de ces modèles, le lecteur peut se référer à ROLAND \& ARDHUIN (2014) et CHAILAN (2015). Afin de comparer les résultats de ces modèles, nous avons utilisé les données du Centre d'Archivage National de Données de Houle In Situ (CANDHIS), prétraitées et mises à disposition par le CEREMA. Les 16 sites sélectionnés correspondent aux stations de suivi à long-terme déployées le long des côtes françaises, ayant enregistré des données avant 2012 (figure 1). Les paramètres étudiés sont la hauteur significative des vagues $\left(\mathrm{H}_{\mathrm{s}}\right)$, la direction moyenne $\left(\theta_{\mathrm{m}}\right)$ et la période moyenne $\left(\mathrm{T}_{02}\right)$. Trois paramètres statistiques ont été retenus pour valider les performances des modèles : le biais (moyenne modèles - moyenne données) normalisé par la moyenne des données, l'erreur quadratique moyenne normalisée par la moyenne des données (EQMN) et le coefficient de corrélation au carré (aussi appelé coefficient de détermination, $\mathrm{R}^{2}$ ). Pour la direction moyenne des vagues, nous avons utilisé le biais et l'erreur quadratique moyenne $(\mathrm{EQM})$. Ces statistiques ont été calculées sur des valeurs moyennées sur 2 heures. 


\section{Thème 1 - Hydrodynamique côtière}

\section{Résultats}

\subsection{Validation}

La figure 3 représente les erreurs statistiques associées aux comparaisons entre les modèles et les observations, pour les paramètres $\mathrm{H}_{\mathrm{s}}$, $\mathrm{T}_{02}$, et $\theta_{\mathrm{m}}$. Pour $\mathrm{H}_{\mathrm{s}}$, les biais normalisés sont compris entre $-30 \%$ et $10 \%$, les EQMN sont comprises entre $12 \%$ et $45 \%$, et les $\mathrm{R}^{2}$ sont compris entre 0.79 et 0.96 . En moyenne, les performances des modèle sont bonnes (biais normalisé $=9.3 \%$, EQMN $=23.6 \%, \mathrm{R}^{2}=0.90$ ), mais on observe toutefois des différences importantes entre les résultats du modèle Atlantique $\left(\mathrm{NB}=4.6 \%, \mathrm{EQMN}=16.5 \%, \mathrm{R}^{2}=0.94\right)$ et du modèle Méditerranée (biais normalisé $=19.5 \%, \mathrm{EQMN}=39.3 \%, \mathrm{R}^{2}=0.82$ ). On peut aussi noter que le modèle Méditerranée sous-estime significativement les $\mathrm{H}_{\mathrm{s}}$.
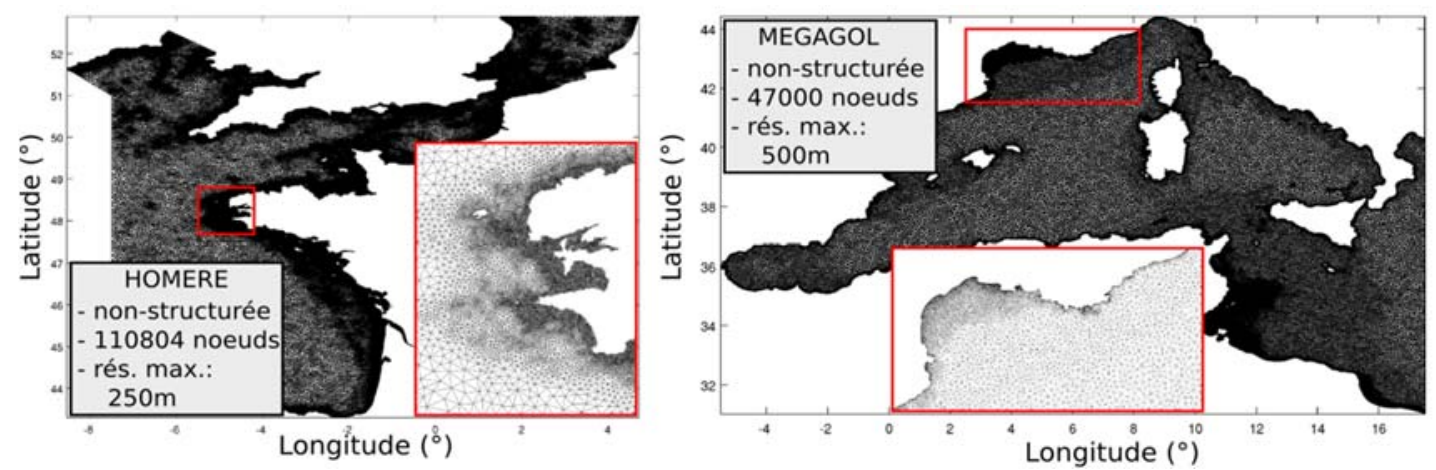

Figure 2. Maillages non-structurés des rejeux HOMERE, sur la façade Atlantique (à gauche) et MEGAGOL, sur la façade Méditerranéenne (à droite).

Pour $\mathrm{T}_{02}$, les biais normalisés sont compris entre $-19 \%$ et $3 \%$, les EQMN sont comprises entre $10 \%$ et $27 \%$ et les $\mathrm{R}^{2}$ sont compris entre 0.27 et 0.83 . Pour ce paramètre également, les performances du modèles sont bonnes en moyenne (biais normalisé $\left.=8.3 \%, \mathrm{EQMN}=17.6 \%, \mathrm{R}^{2}=0.65\right)$ mais avec des disparités fortes entre Atlantique $\left(\mathrm{NB}=4.6 \%, \mathrm{EQMN}=14.8 \%, \mathrm{R}^{2}=0.70\right)$ et Méditerranée $(\mathrm{NB}=-14.7 \%$, $\left.\mathrm{EQMN}=23.7 \%, \mathrm{R}^{2}=0.57\right)$. On notera cependant que les performances du modèle Atlantique sont nettement inférieures dans la Manche avec les coefficients de déterminations les plus bas aux bouées Paluel $\left(R^{2}=0.42\right)$ et Cherbourg $\left(R^{2}=0.27\right)$.

Pour $\theta \mathrm{m}$, les biais sont compris entre $-7^{\circ}$ et $12^{\circ}$, et les EQM sont comprises entre $8^{\circ}$ et $45^{\circ}$. Encore une fois, les performances sont relativement bonnes en moyennes (biais $=$ $4^{\circ}$ et $\mathrm{EQM}=24^{\circ}$, avec toutefois des erreurs plus importantes avec le modèle Méditerranée (biais $=6^{\circ}, \mathrm{EQM}=38^{\circ}$ ) qu'avec le modèle Atlantique (biais $=3^{\circ}, \mathrm{EQM}=$ $17^{\circ}$ ). Ces différences entre les performances des deux modèles s'expliquent essentiellement par les champs de vents utilisés et l'ajustement des paramètres du modèle (RASCLE \& ARDHUIN, 2013). 


\section{XVèmes Journées Nationales Génie Côtier - Génie Civil \\ La Rochelle, 29 au 31 mai 2018}

\subsection{Climat de vagues}

Afin d'étudier la variabilité spatiale du climat de vague le long du littoral français, nous avons représenté sur la figure 4 les valeurs moyennes annuelles, hivernales (DJFM) et estivales (JJAS) des paramètres $\mathrm{H}_{\mathrm{s}}, \mathrm{T}_{02}$ et $\theta_{\mathrm{m}}$ pour 114 points du modèle se trouvant sur l'isobathe $30 \mathrm{~m}$ et espacés d'une vingtaine de kilomètres (cf. figure 1). Cette figure permet d'identifier plusieurs zones aux régimes de vagues bien distincts. De Calais à Cherbourg, les statistiques sont relativement homogènes, avec des $\mathrm{H}_{\mathrm{s}}$ annuelles inférieures à $1.5 \mathrm{~m}$, des $\mathrm{T}_{02}$ inférieures à $4 \mathrm{~s}$ et des $\theta_{\mathrm{m}}$ de secteur NO. De Cherbourg à Saint Nazaire, les statistiques sont très hétérogènes, du fait du découpage fortement marqué du littoral et de la présence de nombreuses îles à proximité des côtes. Les $\mathrm{H}_{\mathrm{s}}$ annuelles varient entre $0.5 \mathrm{~m}$ et $2.5 \mathrm{~m}$, les $\mathrm{T}_{02}$ annuelles varient entre $3 \mathrm{~s}$ et $7 \mathrm{~s}$ et les $\theta_{\mathrm{m}}$ annuelles varient du secteur SO (au sud de la Bretagne) au secteur NO (au nord de la

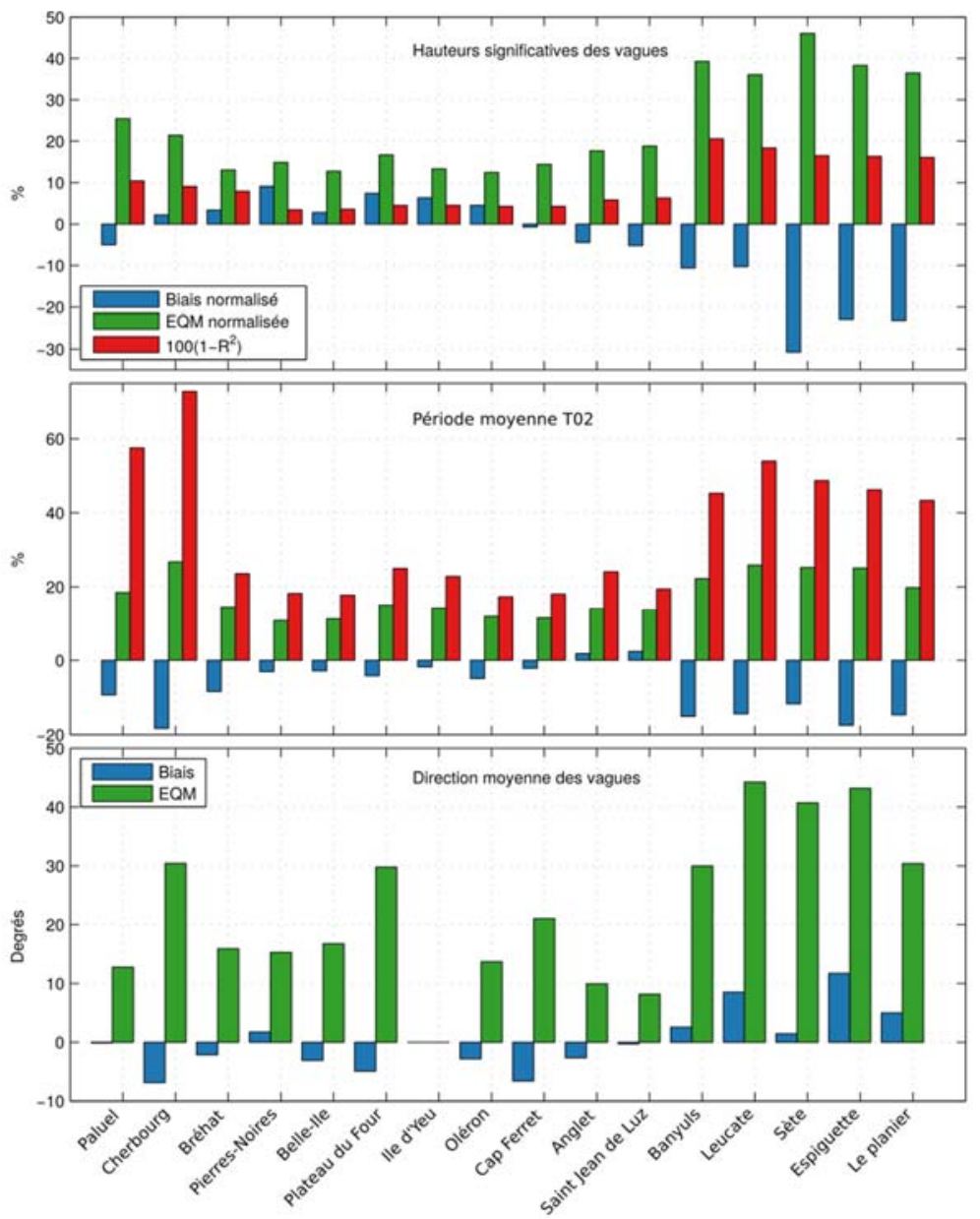

Bretagne).

Figure 3. Biais normalisés, erreurs quadratiques moyennes normalisées (EQMN) et coefficients de détermination $R^{2}$ (sous la forme $100\left(1-R^{2}\right)$ ) pour $H_{s}$ (en haut) et $T_{02}$ (au milieu). Biais et erreurs quadratiques moyennes (EQM) pour $\theta_{m}$ (en bas). 


\section{Thème 1 - Hydrodynamique côtière}

De Saint-Nazaire à Bayonne, les statistiques sont de nouveaux relativement homogènes et représentatives d'un climat dominé par les houles océaniques, avec des $\mathrm{H}_{\mathrm{s}}$ annuelles supérieures à $1.5 \mathrm{~m}$, des $\mathrm{T}_{02}$ annuelles supérieures à $5 \mathrm{~s}$ et des $\theta_{\mathrm{m}}$ annuelles de secteurs OSO à ONO. En Méditerranée, les hauteurs de vagues et périodes moyennes annuelles sont plus faibles que sur le reste du littoral français $\left(\mathrm{H}_{\mathrm{s}}<1 \mathrm{~m}\right.$ et $\mathrm{T}_{02}<4$ s), et les directions des vagues sont comprises entre les secteurs ENE et SSO.

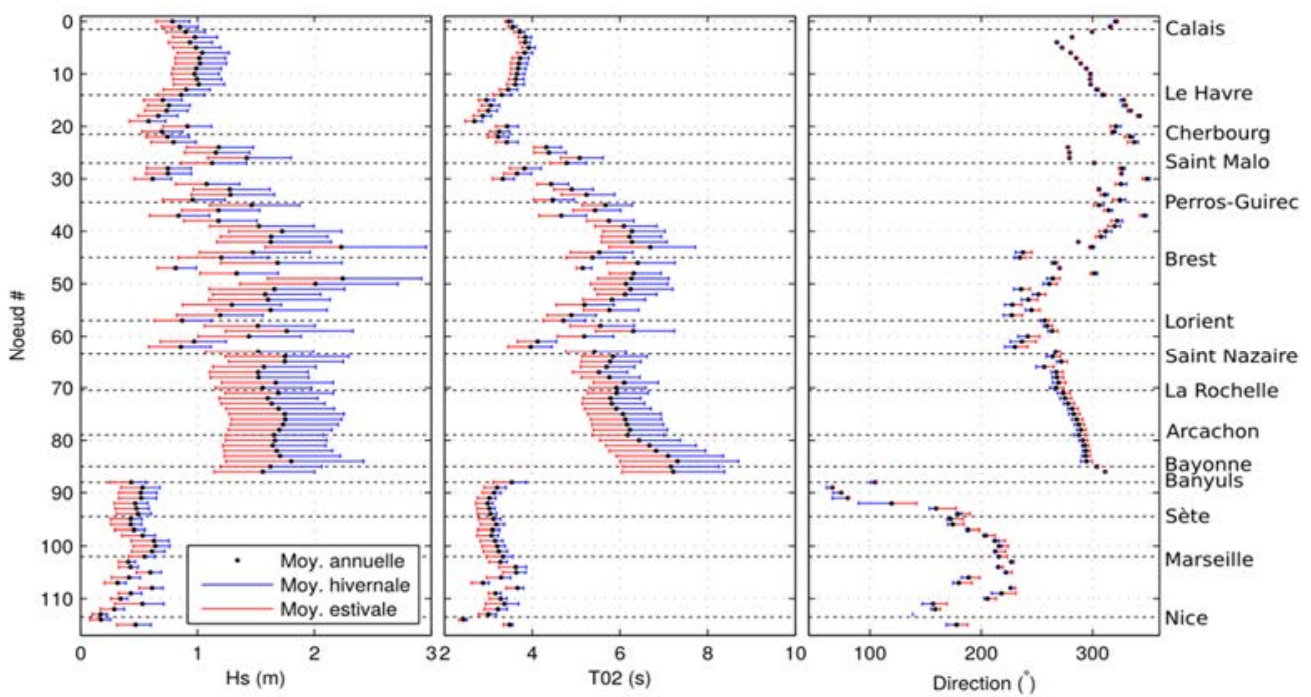

Figure 4. Moyennes annuelles, estivales (JJAS) et hivernales (DJFM) des hauteurs significatives (à gauche), périodes moyennes (au milieu) et directions moyennes (à droite) des vagues le long du littoral de France métropolitaine.

L'amplitude des variations saisonnières présente également une forte disparité spatiale. Les plus fortes variations saisonnières de $\mathrm{H}_{\mathrm{s}}$ et $\mathrm{T}_{02}$ sont observées le long des côtes les plus exposés de la mer d'Iroise et du Golfe de Gascogne. Au contraire, en Manche et en Méditerranée, la saisonnalité de $\mathrm{H}_{\mathrm{s}}$ et $\mathrm{T}_{02}$ est moins marquée. En effet, en Manche comme en Méditerranée, les mers de vent dominent plus fortement les états de mer (d'où les faibles valeurs de $\mathrm{T}_{02}$ ). Or la variabilité saisonnière des paramètres de vagues est fortement contrôlée par la variabilité saisonnière de la circulation atmosphérique, qui elle influence essentiellement les houles océaniques. On note cependant une différence importante entre la saisonnalité des directions de vagues, quasi-inexistante en Manche et nettement plus marquée en Méditerranée. En été, les trajectoires des dépressions de l'Atlantique sont décalées vers le nord et n'atteignent plus la Méditerranée. Le régime de vagues y est alors essentiellement contrôlé par les phénomènes atmosphériques locaux, pouvant seuls expliquer la plus forte variabilité saisonnière de la direction incidente des vagues. 


\section{XVèmes Journées Nationales Génie Côtier - Génie Civil La Rochelle, 29 au 31 mai 2018}

Finalement, nous avons analysé les tendances en $\mathrm{H}_{\mathrm{s}}$ le long du littoral français sur les 18 années (1994-2012) couvertes par les rejeux de paramètres de vagues (figure 5). Ces tendances sont négatives le long de la façade Atlantique alors qu'elles sont positives le long de la façade Méditerranéenne. Il faut bien noter que la variabilité pluriannuelle du climat de vagues est très fortement contrôlée par les oscillations de la circulation atmosphérique représentée par des indices climatiques tels que l'Oscillation Nord Atlantique (ONA) ou l'indice West Europe Pressure Anomaly (WEPA) décrit par CASTELLE et al. (2017). Sur la période 1994-2012, les indices ONA et WEPA présentent une tendance négative $(-0.0001$ et -0.0003 respectivement). Or on observe que les moyennes hivernales de $\mathrm{H}_{\mathrm{s}}$ sont corrélées positivement avec les indices ONA et WEPA sur la façade Atlantique, alors qu'elles sont corrélées négativement avec l'indice ONA sur la façade Méditerranéenne. Ainsi, les tendances observées sur ces deux façades maritimes s'expliquent principalement par des modulations pluriannuelles de la circulation atmosphérique dans l'Atlantique Nord.
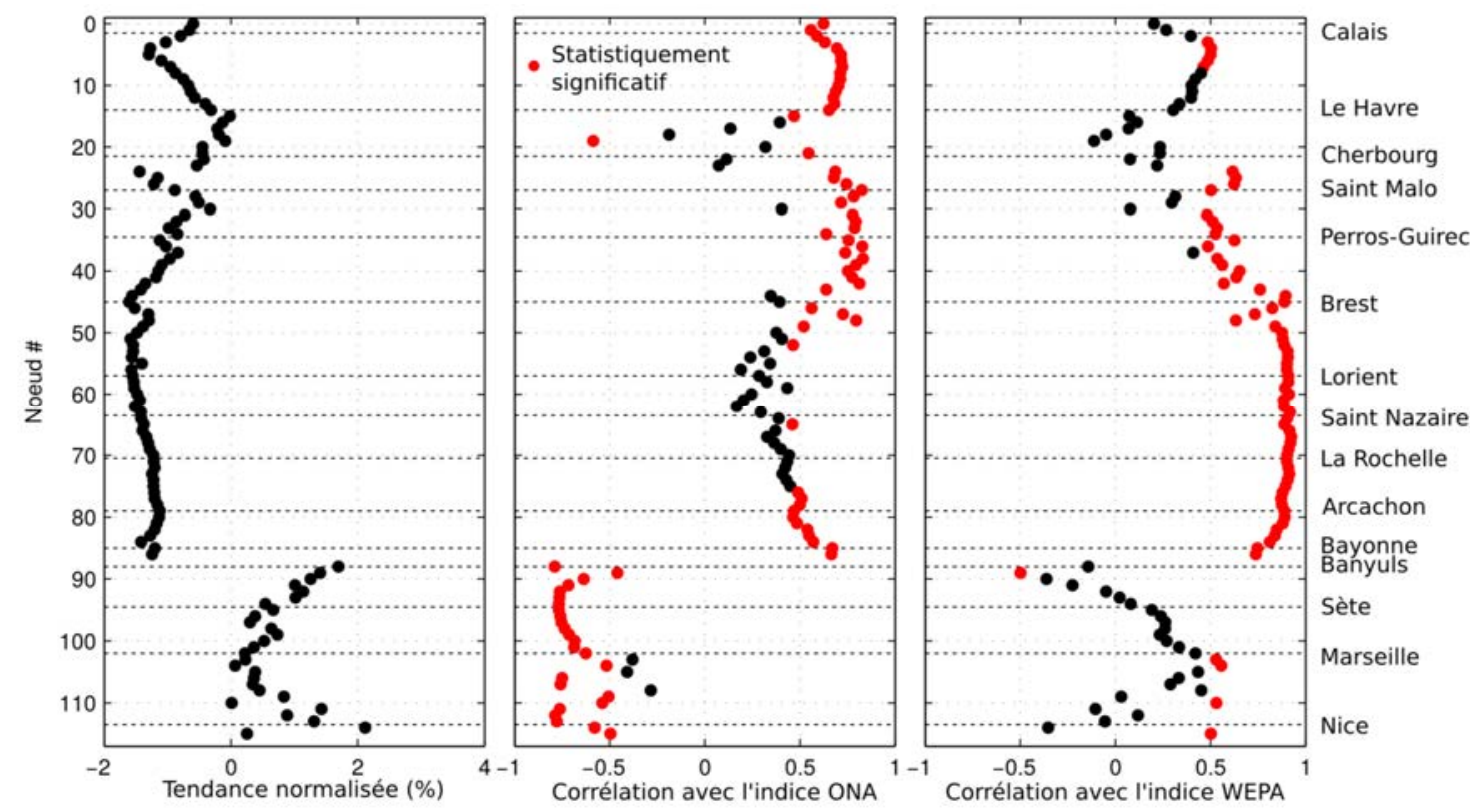

Figure 5. Tendances des moyennes hivernales de hauteurs significatives des vagues sur la période 1994-2012 (à gauche), corrélations des moyennes hivernales de hauteurs significatives des vagues avec l'indice ONA (au milieu) et avec l'indice WEPA (à droite) le long du littoral de France métropolitaine.

\section{Conclusion}

Dans cette étude, nous avons analysé une base de données de paramètres de vagues le long des côtes françaises métropolitaines sur la période 1994-2012. Ces données sont issues de deux simulations rétrospectives réalisées avec le modèle spectral WAVEWATCHIII implémenté sur des grilles non-structurées haute résolution pour 


\section{Thème 1 - Hydrodynamique côtière}

l'Océan Atlantique et la Mer Méditerranée. Les comparaisons des sorties de modèle avec des données in-situ ont révélé de bonnes performances des modèles avec toutefois des erreurs plus importantes en Méditerranée. Ces erreurs sont probablement liées au forçage atmosphérique et à la paramétrisation utilisée dans le modèle Méditerranée, et nécessitent d'être étudiées plus en détail. L'analyse des moyennes annuelles et saisonnières a permis de mettre en avant la forte diversité des régimes de vagues le long du littoral métropolitain, qui s'explique par le découpage côtier et les différents forçages atmosphériques impliqués dans la génération de mers de vent. Finalement, les tendances observées sur la période 1994-2012 sont essentiellement contrôlées par les fluctuations pluriannuelles de la circulation atmosphérique dans l'océan Atlantique Nord.

\section{Références bibliographiques}

BENOIT M., MARCOS F., BECQ F. (1996). Development of a third generation shallow-water wave model with unstructured spatial meshing. Coastal Engineering Proceedings, pp 465-478.

BENOIT M., LAFON F. (2004). A Nearshore wave atlas along the coasts of France based on the numerical modeling of wave climate over 25 years. Proceedings of the Coastal Engineering Conference, pp 714-726.

BEUVIER J., SEVAULT F., HERRMANN M., KONTOYIANNIS H., LUDWIG W., RIXEN M., STANEV E., BÉRANGER K., SOMOT S. (2010). Modeling the Mediterranean Sea interannual variability during 1961-2000: Focus on the Eastern Mediterranean Transient. Journal of Geophysical Research. https://doi.org/10.1029/2009JC005950

BOUDIÈRE E., MAISONDIEU C., ARDHUIN F., ACCENSI M., PINEAUGUILLOU L., LEPESQUEUR J. (2013). A suitable metocean hindcast database for the design of Marine energy converters. International Journal of Marine Energy, Special Issue, Selected Papers, EWTEC2013 3-4, e40-e52. https://doi.org/10.1016/j.ijome.2013.11.010

CASTELLE B., DODET G., MASSELINK G., SCOTT T. (2017). A new climate index controlling winter wave activity along the Atlantic coast of Europe: the West Europe Pressure Anomaly. Geophys. Res. Lett. https://doi.org/10.1002/2016gl072379

CHAILAN R. (2015). Application of scientific computing and statistical analysis to address coastal hazards. Thèse de doctorat de l'Université Montpellier 2.

HERRMANN M.J., SOMOT S. (2008). Relevance of ERA40 dynamical downscaling for modeling deep convection in the Mediterranean Sea. Geophysical Research Letter, Vol. 35, L04607. https://doi.org/10.1029/2007GL032442

LAZURE P., DUMAS F. (2008). An external-internal mode coupling for a 3D hydrodynamical model for applications at regional scale (MARS). Advances in Water Resources, Vol. 31, pp 233-250. https://doi.org/10.1016/j.advwatres.2007.06.010 


\section{XVèmes Journées Nationales Génie Côtier - Génie Civil \\ La Rochelle, 29 au 31 mai 2018}

RASCLE N., ARDHUIN F. (2013). A global wave parameter database for geophysical applications. Part 2: Model validation with improved source term parameterization. Ocean Modelling, Vol. 70, pp 174-188. https://doi.org/10.1016/j.ocemod.2012.12.001

ROLAND A, ARDHUIN F. (2014). On the developments of spectral wave models: numerics and parameterizations for the coastal ocean. Coastal Dynamics, Vol. 64, pp 833-846.

https://doi.org/10.1007/s10236-014-0711-z

SAHA S., et al. (2010). The NCEP Climate Forecast System Reanalysis. Bulletin of the American Meteorological Society, Bulletin of the American Meteorological Society.

https://doi.org/10.1175/2010BAMS3001.1

TOLMAN H. L. - and the WAVEWATCH III Development Group. (2014). User manual and system documentation of WAVEWATCH III version 4.18. NOAA/NWS/NCEP/MMAB Technical Note 316194. 
Thème 1 - Hydrodynamique côtière 